\title{
Physics with Photons at the ATLAS
}

\section{Experiment}

Valeria Perez Reale (CERN) On behalf of the ATLAS Collaboration

Photon 2007, Paris, July 9-13, 2007
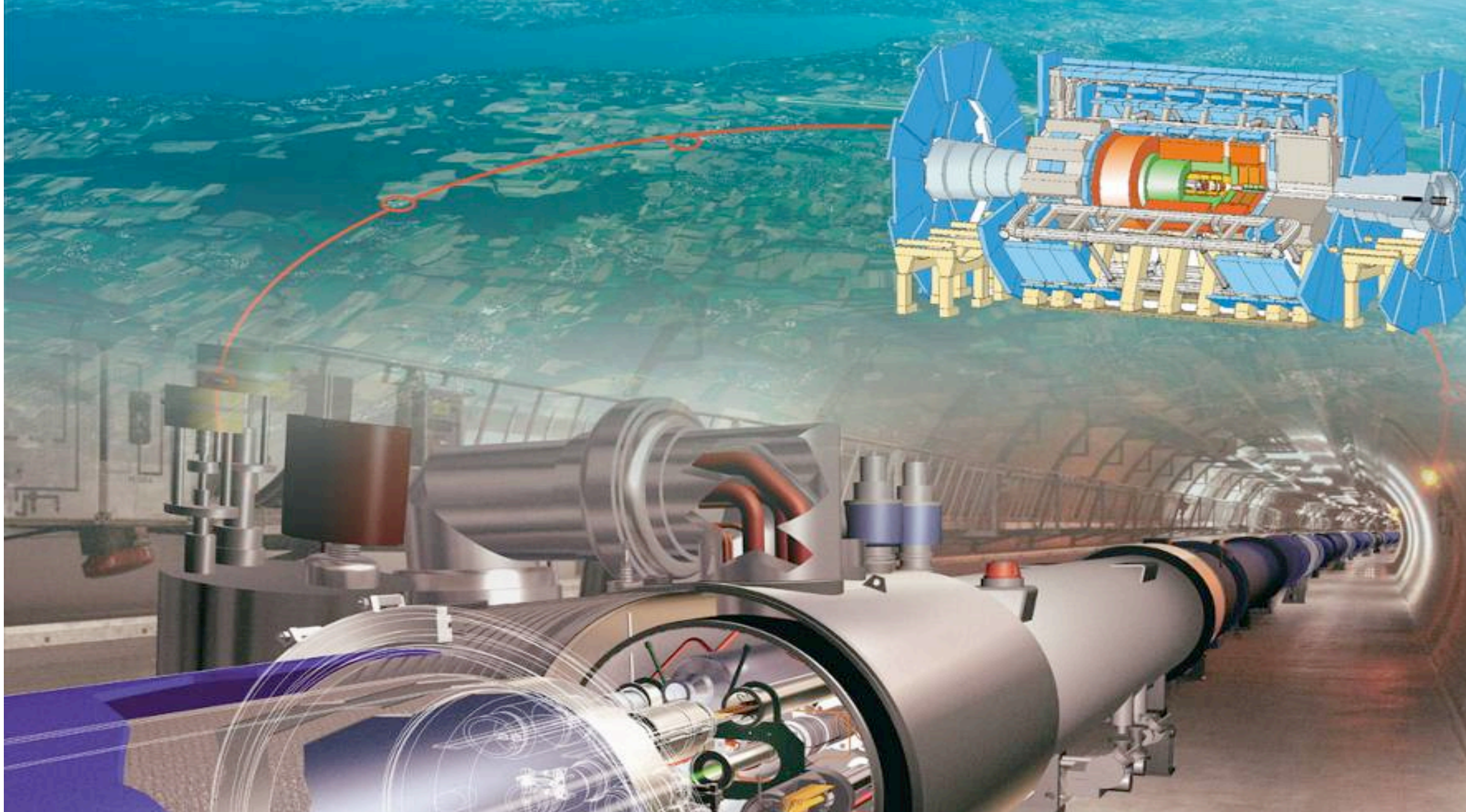


\section{OUTLINE}

(I) Physics Motivation: Single and di-photon direct production at LHC

(II) Detector Performance Requirements: SM Higgs $\mathrm{H} \rightarrow \gamma \gamma$

(III) Experimental Issues: Photon ID

(IV) Experimental Issues: Trigger

(V) Direct Photon Production

(VI) Conclusions and Summary

- Disclaimer: Not all SM and Physics beyond the SM physics signatures with photons are covered in this talk: Wry, black holes, GMSB SUSY Models with non pointing photons, Exotics ... 


\section{(I) LHC: Large Hadron Collider}

- $p p$ collisions at $\sqrt{ }_{\mathrm{s}}=14 \mathrm{TeV}$

- Bunch crossing every 25 ns (40 $\mathrm{MHz}$ )

- Low Luminosity $L=10^{33} \mathrm{~cm}^{-2} \mathrm{~s}^{-1}$ ( $\mathscr{L}=1 \mathrm{fb}^{-1} /$ year)

- High Luminosity $L=10^{34} \mathrm{~cm}^{-2} \mathrm{~s}^{-1}$ ( $L=100 \mathrm{fb}^{-1} /$ year)

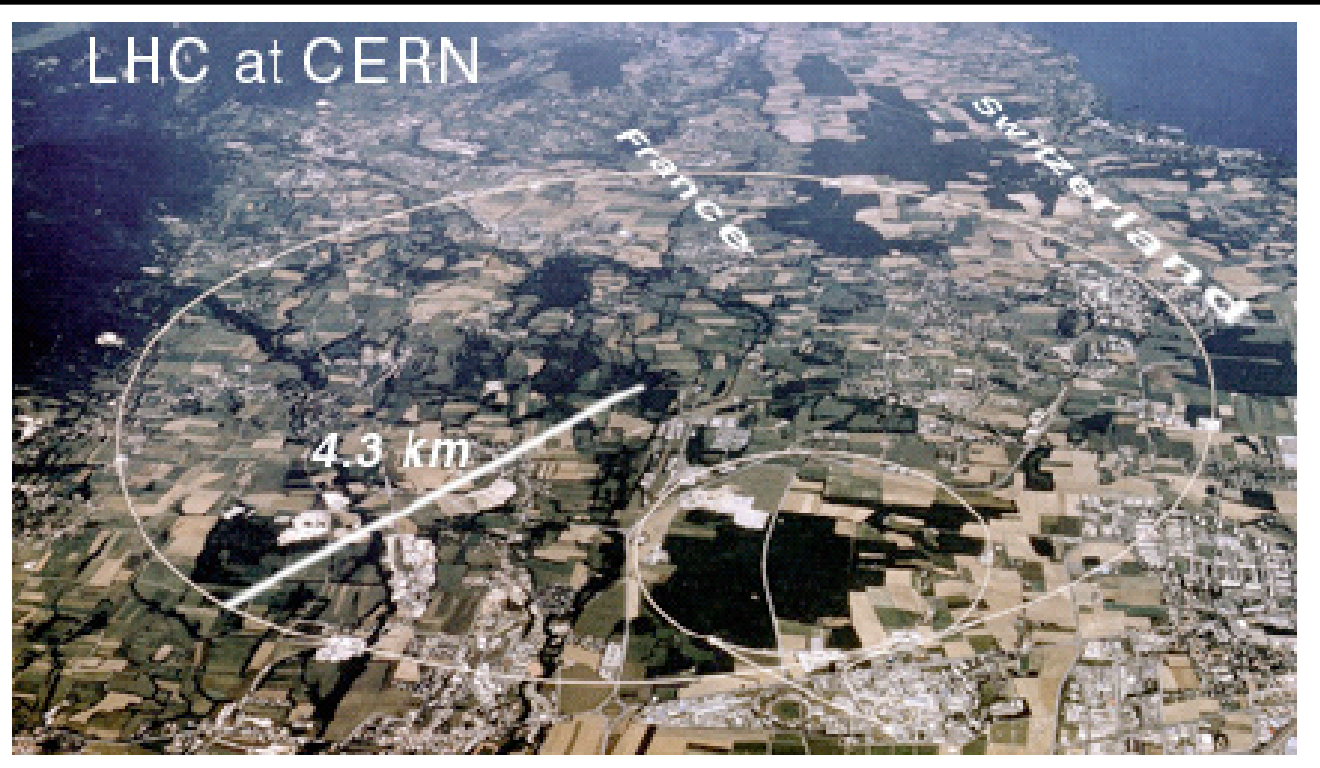

\begin{tabular}{|c|c|c|c|}
\hline Process & Events & $\sigma(\mathrm{nb})$ & $\Rightarrow$ Large statistics: small statistical error \\
\hline & for $10 \mathrm{fb}^{-1}$ & & Production cross section and \\
\hline Inclusive bbbar & $10^{13}$ & $5 \times 10^{5}$ & dynamics largely controlled \\
\hline $\begin{array}{c}\text { Direct photon } \\
\left(p^{\top}>20 \mathrm{GeV}\right)\end{array}$ & $10^{7}$ & 100 & Mass reach up to $\sim 5 \mathrm{TeV}$ \\
\hline $\begin{array}{l}\text { Photon pairs } \\
\left(p_{T}>20 \mathrm{GeV}\right)\end{array}$ & $10^{4}$ & 15 & $\begin{array}{l}\text { Test QCD predictions and } \\
\text { perform precision }\end{array}$ \\
\hline
\end{tabular}


- The study of single photons and di-photons produced in the primary parton-parton interaction (direct photon production) is interesting in itself:

- Their production and associated measurement provides a direct test of perturbative quantum chromodynamics ( $p Q C D$ )

- The coupling to the interaction partons provided by the photon allows the parton content of the proton to be probed directly (provides a possible constraint on the gluon content of the proton)

- The topology of events with photons recoiling against a jet allows the hadronic calorimeter to be calibrated with the electromagnetic calorimeter using energy balance in the event.

- High $\mathrm{p}_{\mathrm{T}}$ single photons and photon pairs are important for the discovery of many Standard Model and "beyond the Standard Model processes", the measurement and understanding of direct photon production are essential for the search of new physics:

- SM Higgs channel into $\gamma$

- Exotics di-photon production and SUSY physics 


\section{(II) Detector Performance Requirements}

- $\mathrm{H} \rightarrow \gamma \gamma$ is a rare decay mode with $\mathrm{BR} \sim 10^{-3}$

- The signal should be visible as a small peak above the $\gamma \gamma$ continuum background: need $\sigma$ $(\mathrm{m}) / \mathrm{m} \sim 1 \%$

- Good energy resolution and uniformity of the EM calorimeter

- Irreducible background consists of genuine photons pairs continuum.

- Reducible background comes from jet-jet and gamma-jet events in which one or both jets are misidentified as photons (Reducible / irreducible cross section (LO) $\sim 2 \times 10^{6}(\mathrm{jj})$ and $\left.\sim 8 \times 10^{2}(\gamma \mathrm{j})\right)$

- Excellent $\gamma / j e t$ and $\gamma / \pi 0$ separation needed

- Conversion recovery needed

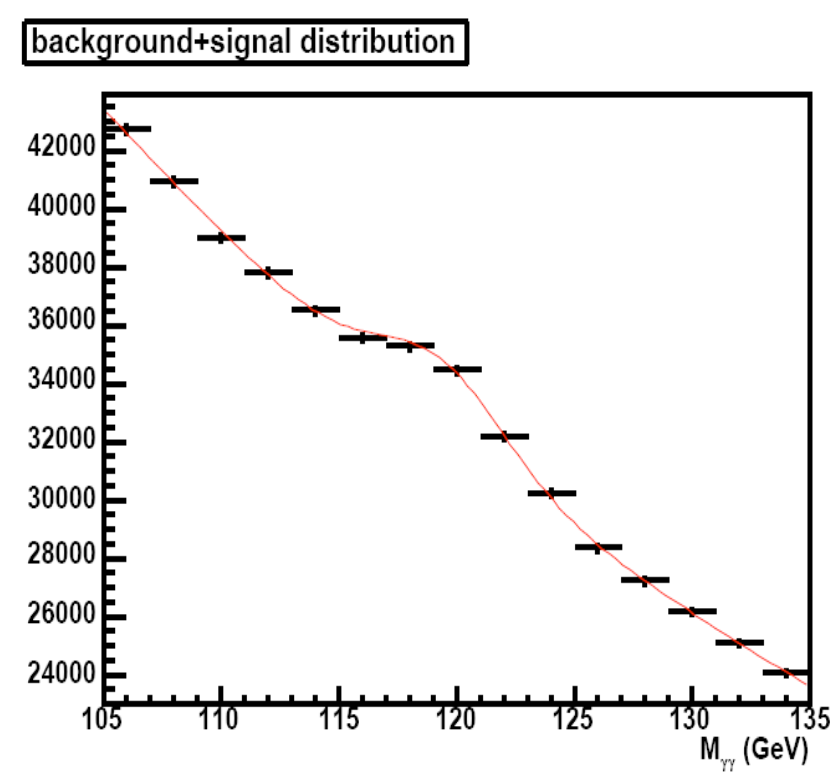

$\gamma$-ID requirements

$\checkmark$ Trigger Efficiency

$\checkmark$ Understanding of detector (alignment and material)

$\checkmark$ ECAL calibration 


\section{(II)The ATLAS Detector}

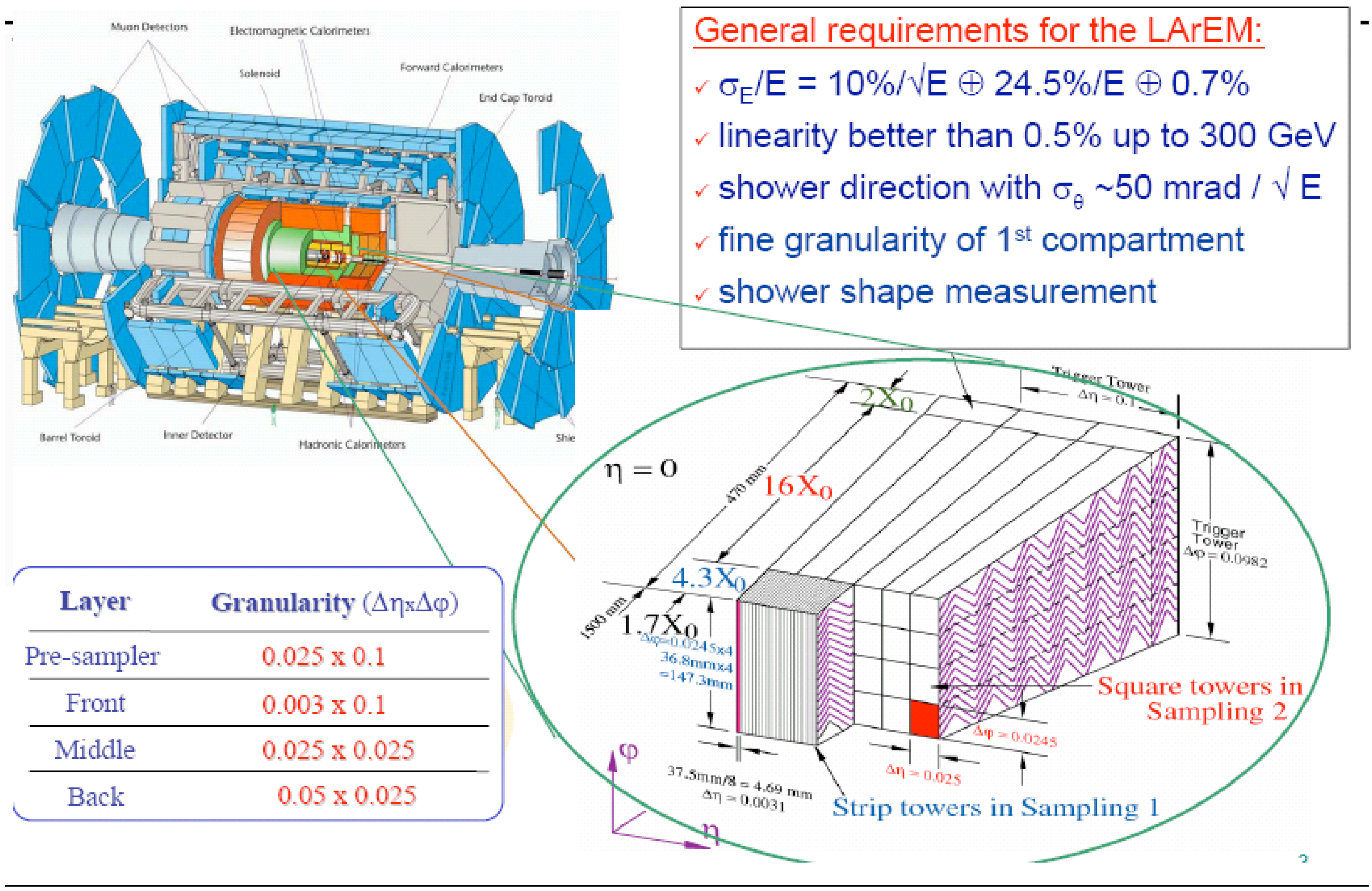




\section{(III) Basis of $\gamma /$ jet and $\gamma / \pi 0$ separation}

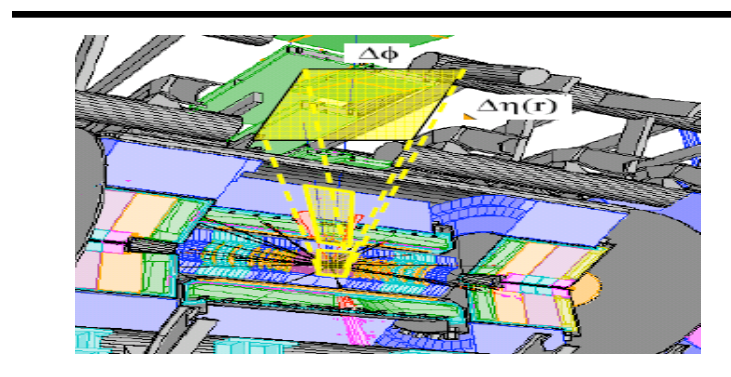

L1 Trigger: EM candidate
EM and HAD isolation
coarse granularity

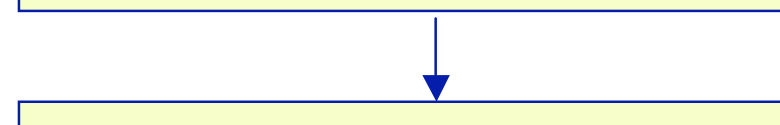

High Level Trigger $\gamma$. confirms L1 decision with more refined granularity

Offline Analysis $\gamma$ : more up to date calibration. Conversion recovery and track veto (use of ID info)
$\gamma-I D$

Leakage in Hadronic calorimeter

EM sampling 2: different transverse development of electromagnetic and hadronic showers.

- shower shapes in $\eta$ and $\varphi$

- shower width in $\eta$ direction

EM sampling 1: only jets with a little hadronic activity survive. Fine segmentation of the strips :

- look for substructures in strips

- shower width in $\eta$

$\eta$ dependent photon identification selection 


\section{(III) $\gamma / \pi 0$ separation}

- After the application of hadronic leakage and 2nd EM sampling criteria, $\sim 80 \%$ of the remaining background is composed of isolated $\pi^{0}$ from jet fragmentation

- The high granularity of the $1^{\text {st }} \mathrm{EM}$ sampling provides additional rejection

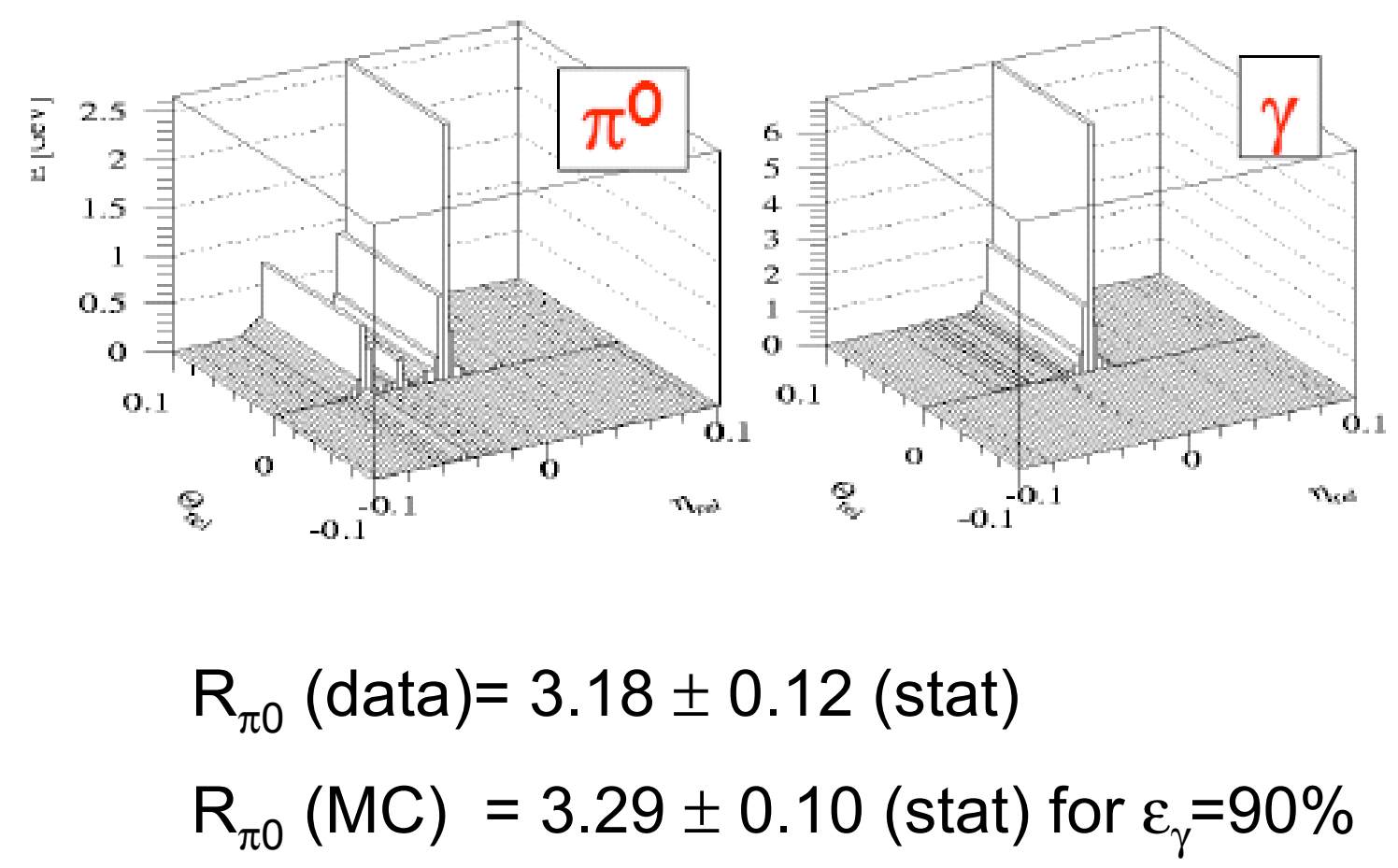

Results from Test Beam $2002 @ 50$ GeV

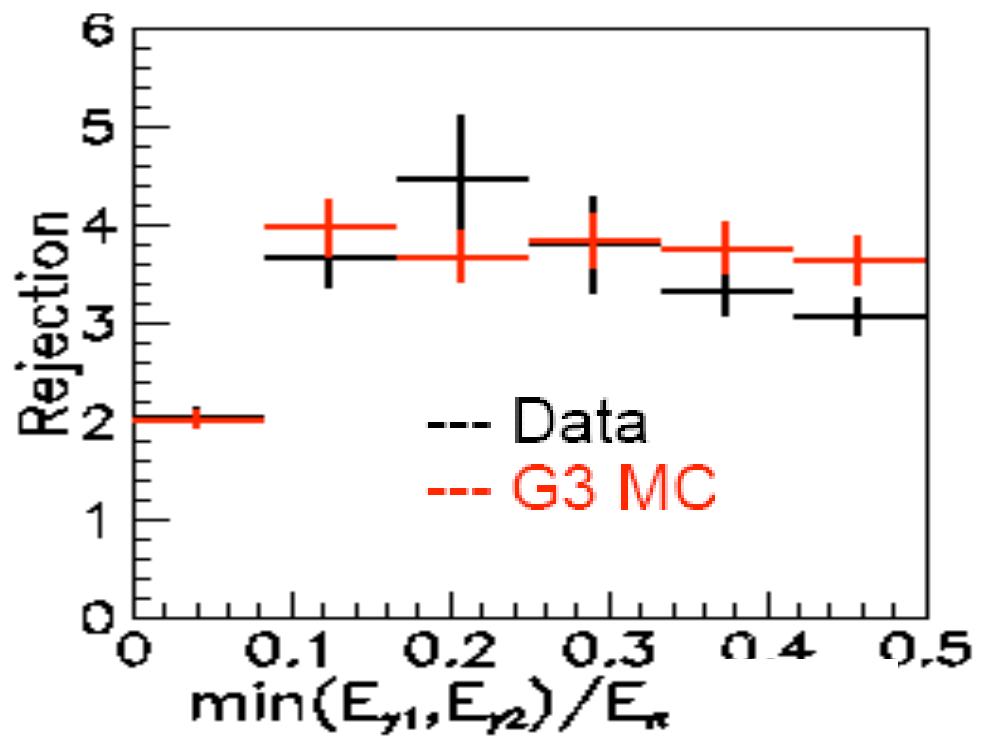




\section{(III) $\gamma /$ jet Separation}

- Performance assessed with single $\gamma$ of different energies or $\gamma$ from $\mathrm{H} \rightarrow \gamma \gamma$

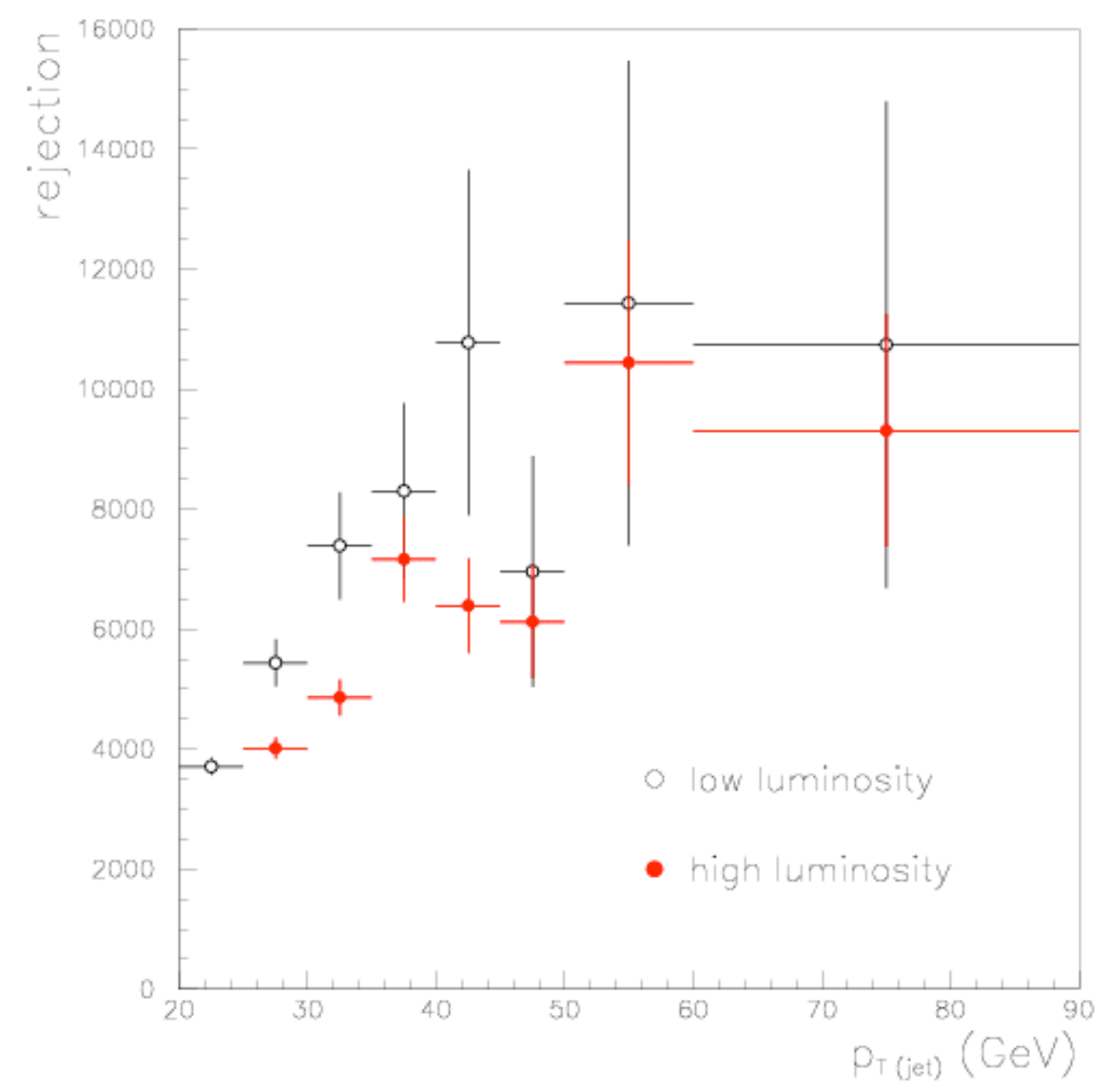

Low luminosity: $2 \times 10^{33} \mathrm{~cm}^{-2} \mathrm{~s}^{-1}$ High luminosity: $1 \times 10^{34} \mathrm{~cm}^{-2} \mathrm{~s}^{-1}$
- For an $\varepsilon \gamma=80 \%$ (flat in $\eta$ and $p_{\mathrm{T}}$ ) a $R_{\text {jet }} \sim \mathbf{5 0 0 0}$ can be achieved for $p_{T}$ $>25 \mathrm{GeV}$

- Looking at the jet origin:

- $R \sim 3 \times 10^{3}$ on quark jets

- $\mathrm{R} \sim 2.1 \times 10^{4}$ on gluon jets

$\rightarrow$ Difference due to softer fragmentation function of gluon jets.

- The reducible background after photon id selection is reduced below the total irreducible background $\gamma \gamma$ 


\section{(IV) Trigger Requirements}

ON-line

OFF-line

- Highly hermetic and granular detectors $\oplus$ large particle multiplicity $\rightarrow$ huge data volume! Average event size 1.5 MB

- 25 ns bunch spacing $\rightarrow$ high event rates! Mass storage $300 \mathrm{MB} / \mathrm{s}$

- At design luminosity pile-up of about $\mathbf{2 3}$ interactions on average per each event

- High energy (14 TeV) $\rightarrow$ Huge QCD backgrounds

- Low cross sections for discovery physics (e.g., Higgs production) $\rightarrow$ Rejection power

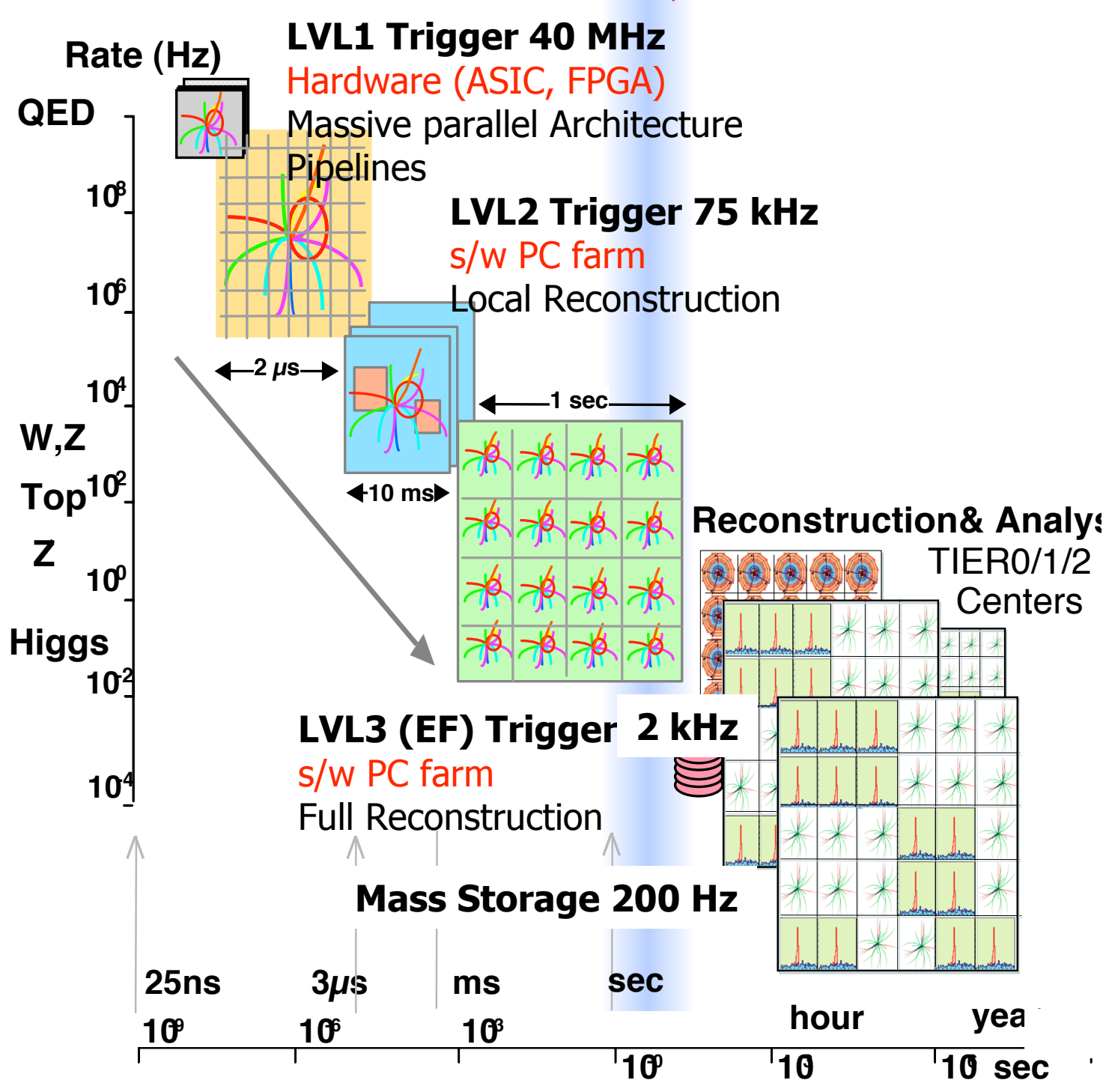




\section{(IV) Trigger: $\gamma /$ jet separation}

- The optimal photon selection efficiency is a compromise between trigger efficiency, event rate allowed (few tens of $\mathrm{Hz}$ out of total $200 \mathrm{~Hz}$ for $L=10^{33} \mathrm{~cm}^{2} \mathrm{~s}^{-1}$ ) and system performance limitations

- For photon target goal is $\mathbf{8 0 \%}$ efficiency after the last trigger selection step (EF) for a rejection factor $R \sim 1000$

- Trigger efficiency normalized wrt offline reconstructed kinematical cuts in $\mathrm{E}_{\mathrm{T}}$ and $|\eta|<2.4$
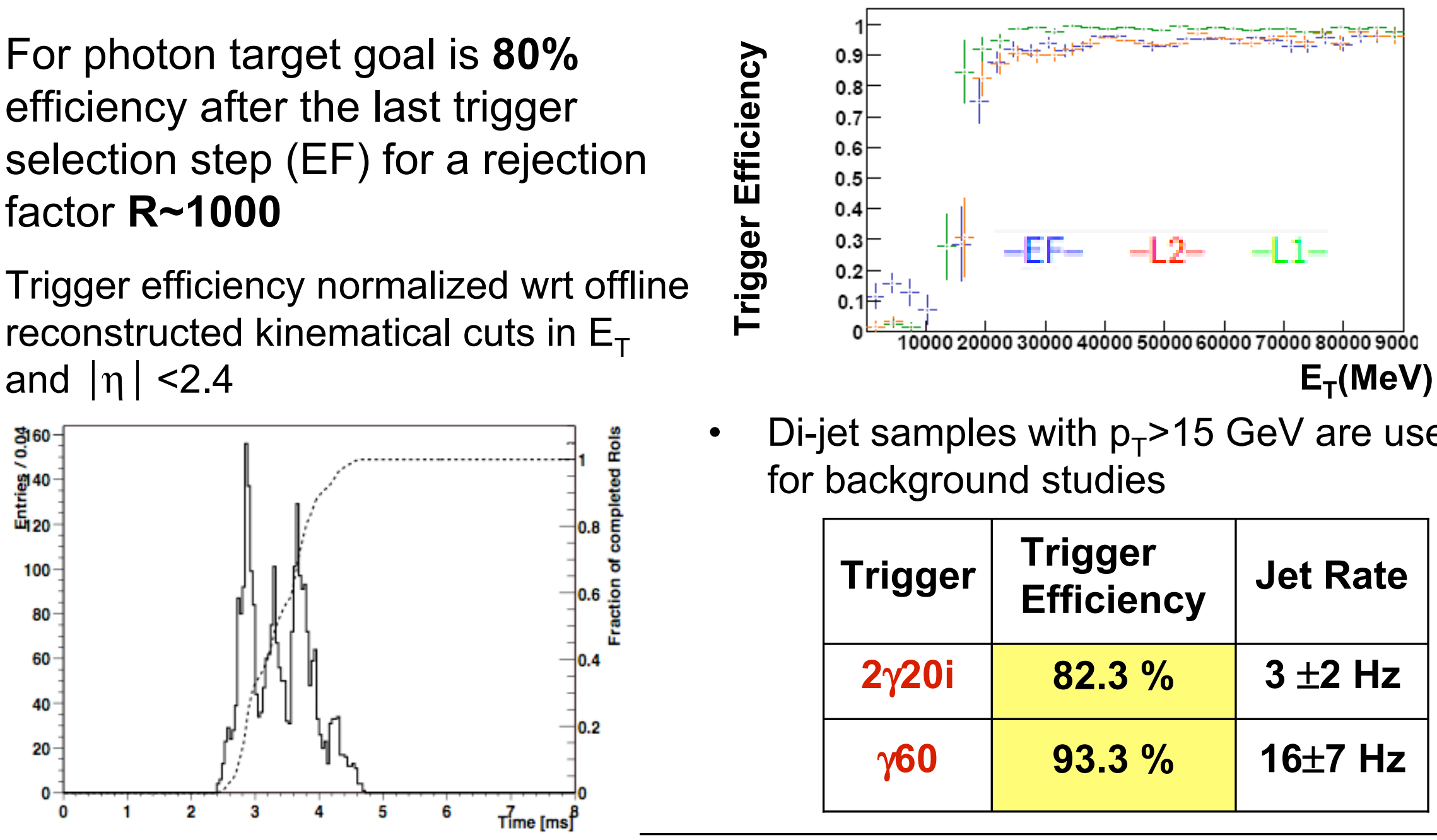

- Di-jet samples with $p_{\mathrm{T}}>15 \mathrm{GeV}$ are used for background studies

\begin{tabular}{|c|c|c|}
\hline Trigger & $\begin{array}{l}\text { Trigger } \\
\text { Efficiency }\end{array}$ & Jet Rate \\
\hline $2 \gamma 20 i$ & $82.3 \%$ & $3 \pm 2 \mathrm{~Hz}$ \\
\hline$\gamma 60$ & $93.3 \%$ & $16 \pm 7 \mathrm{~Hz}$ \\
\hline
\end{tabular}




\section{(IV) Triggering: $\mathrm{H} \rightarrow \gamma \gamma$ Efficiency}

- The $\mathrm{H} \rightarrow \gamma \gamma$ channel can be triggered with an efficiency of $\sim 80 \%$ requiring two isolated photons with $\mathbf{p T}>\mathbf{2 0} \mathbf{~ G e V}$ in the physics precision region of $|\eta|<2.4$ at low luminosity $\mathrm{L}=2 \times 10^{33} \mathrm{~cm}^{2} \mathrm{~s}^{-1}$

\begin{tabular}{|c|c|}
\hline $\begin{array}{l}\text { Trigger } \\
2 \gamma 20 \mathrm{i}\end{array}$ & $\begin{array}{l}\text { Trigger } \\
\text { Efficiency }\end{array}$ \\
\hline L1 & $96.0 \pm 0.8 \%$ \\
\hline L2 & $88.6 \pm 1.3 \%$ \\
\hline EF & $85.4 \pm 1.5 \%$ \\
\hline
\end{tabular}

- The trigger selection is efficiency selecting both converted and non converted photons of a $\mathrm{H} \rightarrow \gamma \gamma$ event

- Non converted: $80.2 \pm 0.1 \%$

- Converted: $78.5 \pm 0.2 \%$

converted $\mathrm{H}-\mathrm{gg}$ Efflclency

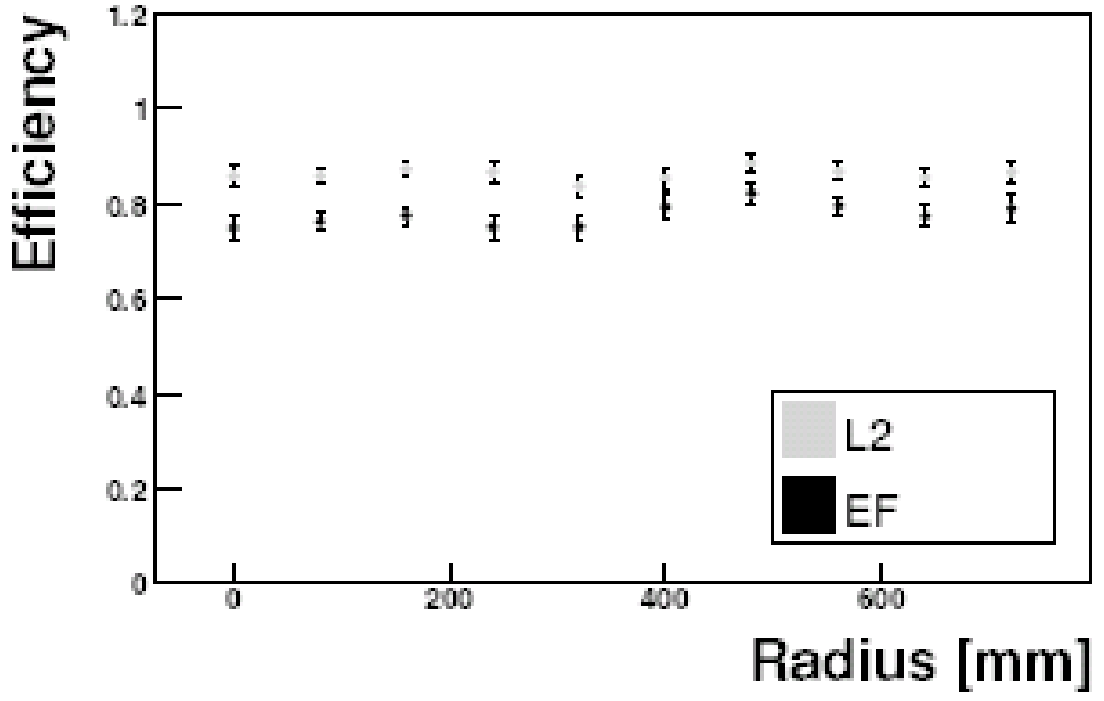

-The addition of pile-up reduces the trigger efficiency $\sim 2 \%(4 \%)$ at low (high) luminosity)

Radius [mm] 


\section{(IV) Triggering: Direct Photon}

- The direct photon can be triggered requiring one photon with $\mathbf{p}_{\mathrm{T}}>\mathbf{2 0} \mathbf{G e V}$ in the "precision physics" region of $|\eta|<2.4$ (simulation results for initial luminosity running $\left.\mathrm{L}=1 \times 10^{31} \mathrm{~cm}^{2} \mathrm{~s}^{-1}\right)$

- Signal sample: $\gamma+$ jet $\left(\gamma\right.$ generated with $\left.p_{T}>10 \mathrm{GeV}\right)$

\begin{tabular}{|c|c|c|}
\hline & $\begin{array}{c}\text { Jet energy } \\
\text { range (GeV) }\end{array}$ & $\begin{array}{c}\text { Trigger } \\
\text { Efficiency\%) } \\
\gamma 20\end{array}$ \\
\hline 1 & $17-35$ & $75.1 \pm 0.3$ \\
\hline 2 & $35-70$ & $83.5 \pm 0.3$ \\
\hline 3 & $70-140$ & $89.3 \pm 0.2$ \\
\hline 4 & $140-280$ & $91.7 \pm 0.2$ \\
\hline 5 & $280-560$ & $94.4 \pm 0.2$ \\
\hline 6 & $560-1120$ & $92.4 \pm 1.1$ \\
\hline
\end{tabular}

- Background from jet-jet sample generated with $p_{T}>15 \mathrm{GeV}$ is $7 \mathrm{~Hz}$ 


\section{(V) Direct Photon Production (LO)}

- Two main contributions (LO):

- $q g \rightarrow \gamma q \quad$ Compton Scattering (dominating)

- $q q \rightarrow \gamma g$ Anhhihilation process

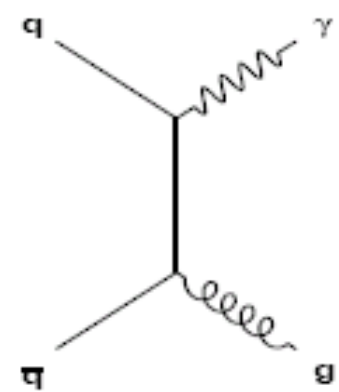

Annihilation Process

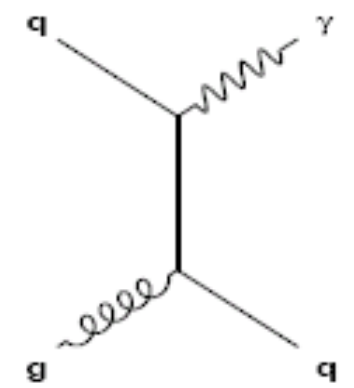

Compton Process

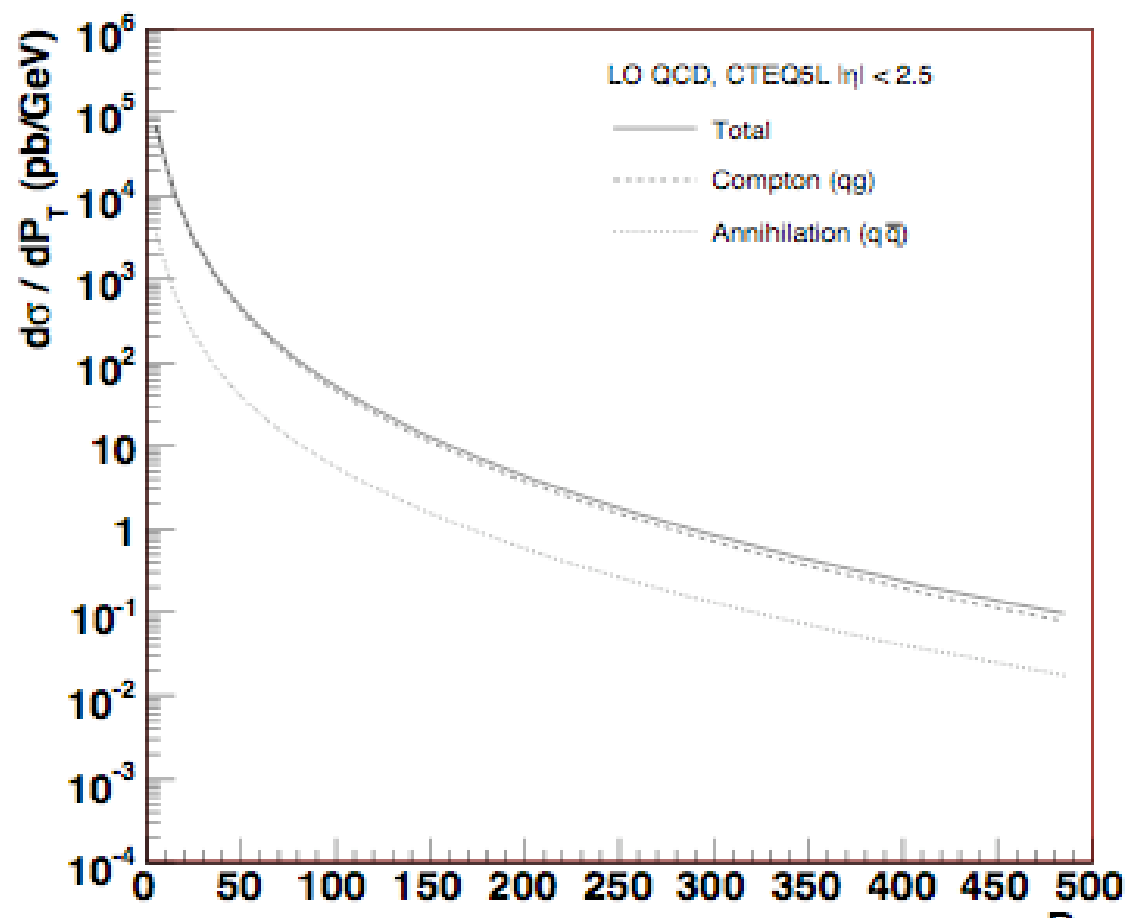

a)

Valeria Perez Reale

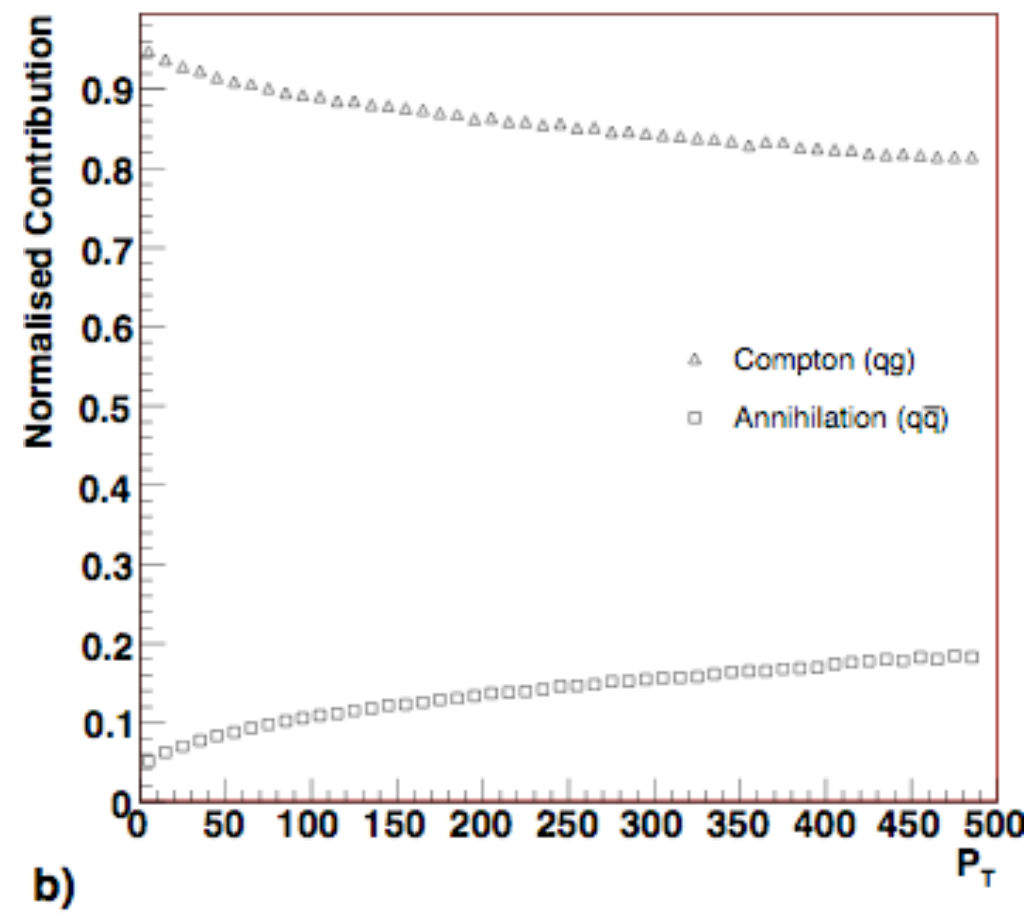




\section{(V) Direct Photon Production (NLO)}
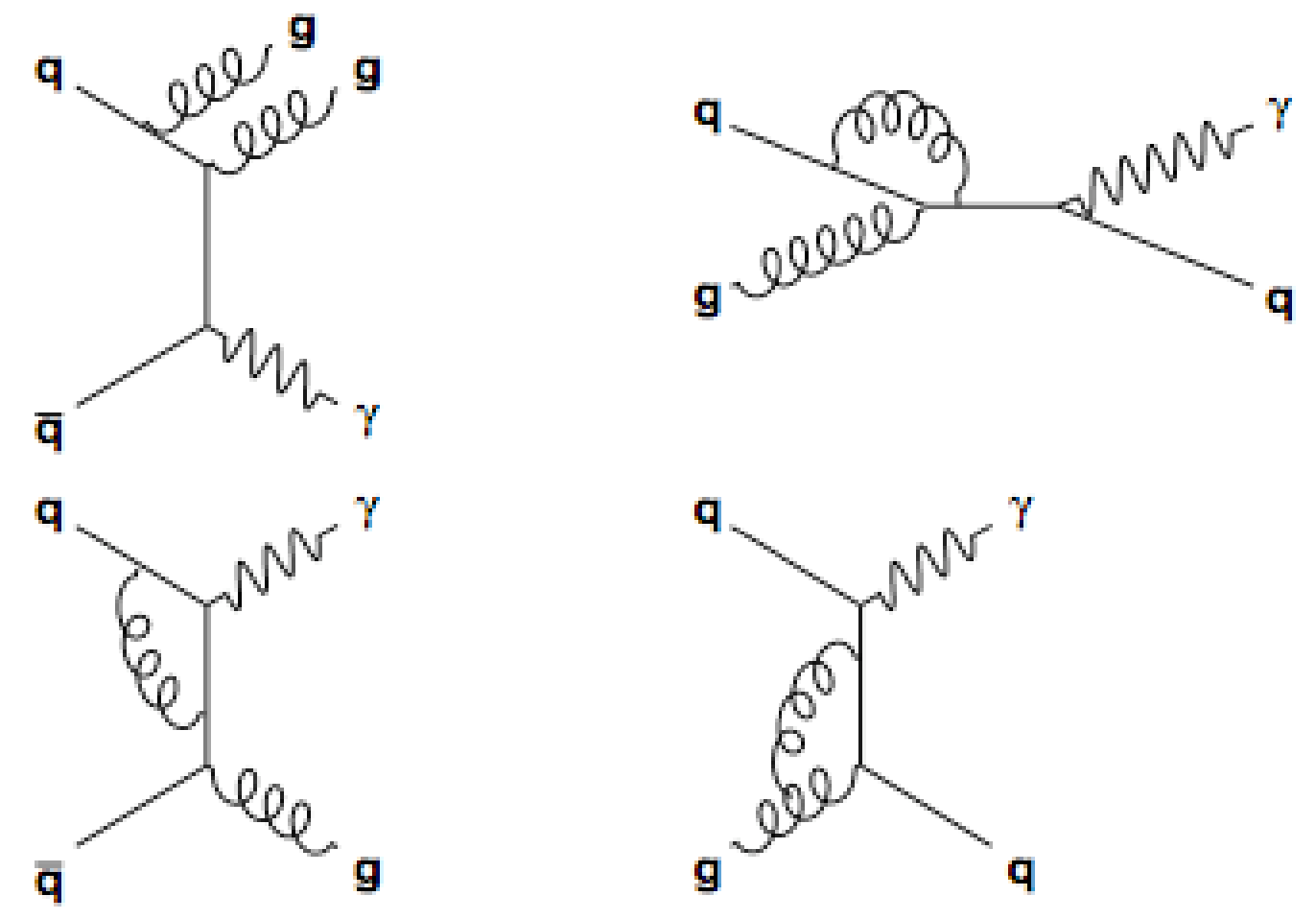

NLO real diagram and corresponding virtual diagrams of Direct Photon Events 


\section{(V) Signal and Background}

- The typical event topology of direct photon production in the ATLAS detector will be the observance of a well-isolated photon recoiling against a jet

- At LO these events should be back-to-back in the $r-\phi$ plane and display a balance of energy between the jet and the photon.

- From QCD it is known that the jet rate will be $\sim 3$ orders of magnitude large than that for direct photons

-The required good photon identification is achieved using the highly-granular

EM calrimeter

- Main background related to fragmentation non perturbative QCD

- The $\pi 0$ s background from jet fragmentation is reduced by requiring a selection in the 1st sampling of EM

- The background is reduced further by requiring an isolation cone (EM, HAD, tracking)

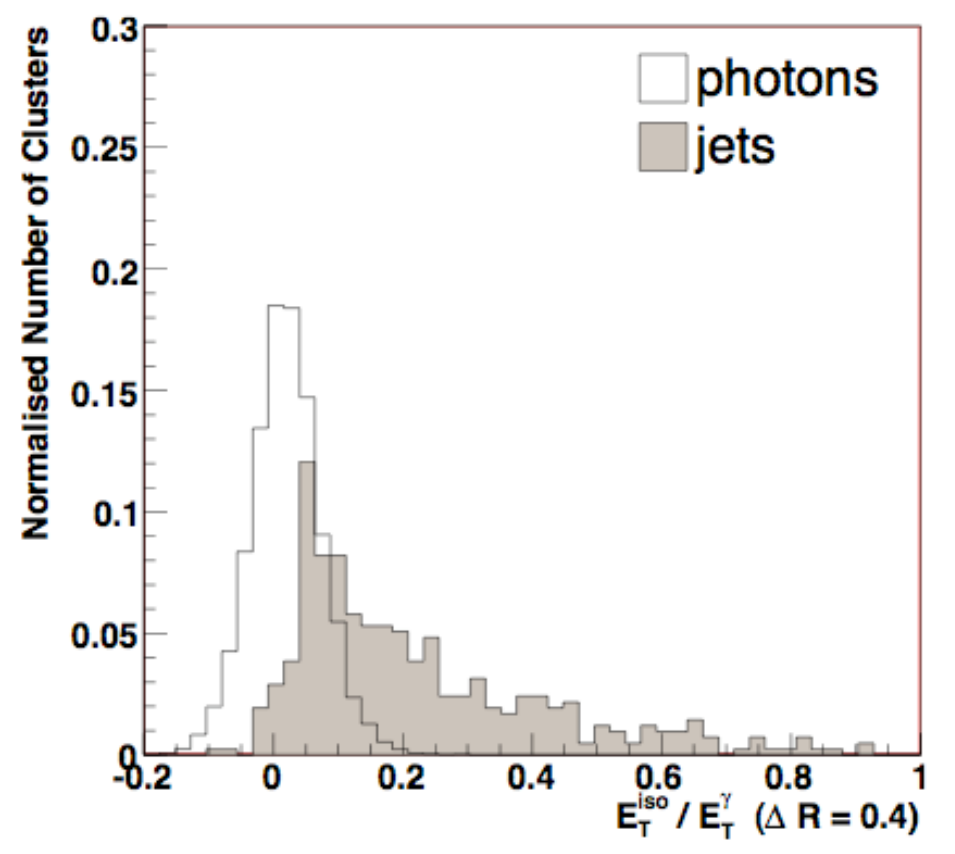




\section{(V) Systematic Errors}

- Sources of experimental systematic errors within the direct photon measurement expected in ATLAS (first approximation):

- Luminosity error: In the initial phase of LHC operation for an integrated luminosity of $1 \mathrm{fb}^{-1}$, the error on the ATLAS measured luminosity $10 \%$ (aim to reduce error significantly, e.g. Using forward detectors)

- Absolute EM energy scale: should be ultimately known $\sim 1 \%$. When convolved with falling $\mathrm{E}_{\mathrm{T}}$ spectrum of direct photons $<5 \%$

- Preselection efficiency: $<5 \%$ for signal and background

- Photon Trigger Efficiency Error: is expected to be $\sim 1 \%$

- Background subtraction provides the other major source of background, a conservative estimate on its effect on the cross section is $10 \%$

- Some of these uncertainties (luminosity, photon trigger efficiency) will cancel out in the S/B measurement contrary to theoretical uncertainties (pdfs, scale variation)

- Aim for a precision on the cross-section determination similar (hopefully better) than observed at D0/CDF ( 15-22\%)

- ATLAS measurement at much higher $\sqrt{ } s$ will extend to very high $p_{T}$ photons 


\section{(V) Constrains on Gluon Structure}

- The kinematic acceptance of the ATLAS detector allows a wide range of $x$ and $Q^{2}$ to be probed $(|y|<5)$

$$
x_{\min }=\frac{x_{T} e^{-\eta}}{2-x_{T} e^{e^{T}}} \quad x_{T}=2 p_{T} / \sqrt{s}
$$

- ATLAS will be sensitive to the gluon fraction $x$ below $10^{-4}$ within an energy scale $\mathbf{Q}^{2}$ above 100 $\mathrm{GeV}^{2}$

- The highest-energy photons will give access to large $\mathbf{x}$ values in the range $\sim 10^{-1}$

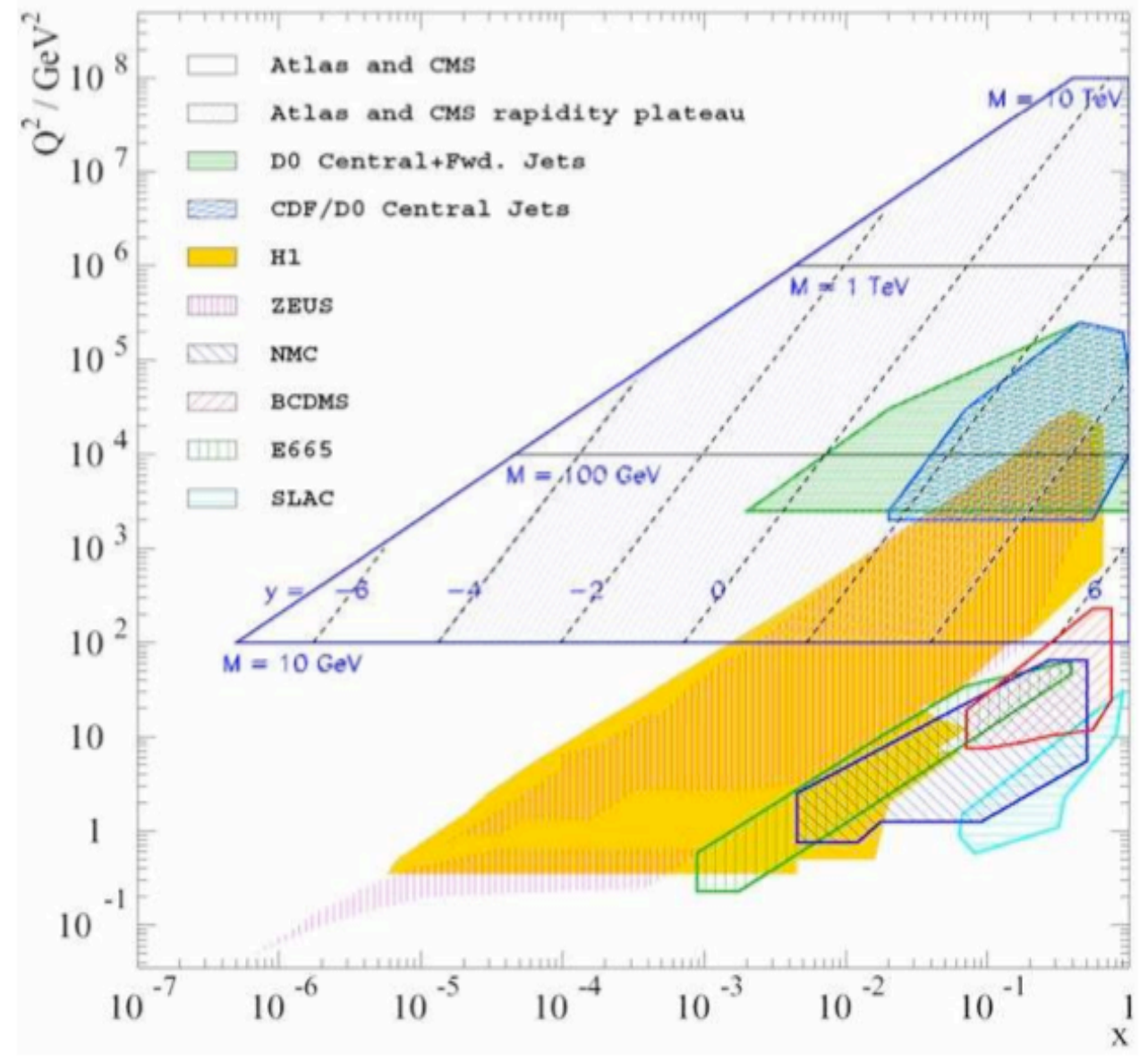




\section{(VI) CONCLUSIONS}

- The LHC will provide large statistics of single and di-photon direct production in the first year of data taking (integrated luminosity of $10 \mathrm{fb}^{-1}$ ) in the energy region $\sqrt{14} \mathrm{TeV}$

- The ATLAS Hadronic and Electromagentic calorimeter has proven to have the $\gamma /$ jet separation and $\gamma / \pi^{0}$ separation capability needed to observe direct photon signal and $\mathrm{SM} \mathrm{H} \rightarrow \gamma \gamma$ over the QCD reducible background

- Jet rejection factor of 5000 for photon efficiency of $80 \%$

- $\pi^{0}$ rejection factor of 3 for photon efficiency of $90 \%$

- ATLAS will measure direct photons at higher $\mathrm{E}_{\mathrm{T}}$ and with a significant improvement in precision and a new $\sqrt{ }_{s}$ wrt to other experiments

- The full potential of the direct photon production process has yet to be realised, the LHC will provide an opportunity to determine the gluon density function in a proton to a new kinematic region of $x\left(\sim 10^{-4}<x<0.1\right)$ and $\mathrm{Q}^{2}\left(10^{2}<\mathrm{Q}^{2}<10^{5} \mathrm{GeV}^{2}\right)$ 


\section{BACK-UP SLIDES}




\section{LHC Machine Parameters}

\begin{tabular}{|l|c|c|c|}
\hline Energy & $\mathrm{E}$ & {$[\mathrm{TeV}]$} & 7.0 \\
Dipole field & $\mathrm{B}$ & {$[\mathrm{T}]$} & 8.4 \\
\hline Luminosity & $\mathrm{L}$ & {$\left[\mathrm{cm}^{-2} \mathrm{~s}^{-1}\right]$} & $10^{34}$ \\
Beam-beam parameter & $\xi$ & & 0.0034 \\
Total beam-beam tune spread & $E_{\mathrm{i}}$ & {$[\mathrm{GeV}]$} & 450 \\
Injection energy & $I_{\mathrm{beam}}$ & {$[\mathrm{A}]$} & 0.53 \\
Circulating current/beam & $k_{\mathrm{b}}$ & & 2835 \\
Number of bunches & $h_{\mathrm{RF}}$ & & 35640 \\
\hline Harmonic number & $\tau_{\mathrm{b}}$ & {$[\mathrm{ns}]$} & 24.95 \\
Bunch spacing & $n_{\mathrm{b}}$ & & $1.0510^{11}$ \\
\hline Particles per bunch & $E_{5}$ & {$[\mathrm{MJ}]$} & 334 \\
\hline Stored beam energy & $\varepsilon_{\mathrm{n}}$ & {$[\mu \mathrm{m} . \mathrm{rad}]$} & 3.75 \\
\hline Normalized transverse emittance $(\beta \gamma) \sigma^{2} / \beta$ & & \\
& & & \\
Collisions & $\beta^{*}$ & {$[\mathrm{~m}]$} & 0.5 \\
$\beta$-value at I.P. & $\sigma^{*}$ & {$[\mu \mathrm{m}]$} & 16 \\
r.m.s. beam radius at. I.P. & $\sigma^{* *}$ & {$[\mu \mathrm{rad}]$} & 32 \\
r.m.s. divergence at I.P. & $L_{\mathrm{b}}$ & {$\left[\mathrm{cm}{ }^{-2}\right]$} & $3.1410^{26}$ \\
Luminosity per bunch collision & $\phi$ & {$[\mu \mathrm{rad}]$} & 200 \\
Crossing angle & $\pi_{\mathrm{b}_{\mathrm{c}}}$ & & 19 \\
\hline Number of events per crossing & $\tau_{\mathrm{beam}}$ & {$[\mathrm{h}]$} & 22 \\
\hline Beam lifetime & $\tau_{L}$ & {$[\mathrm{~h}]$} & 10 \\
Luminosity lifetime & & \\
\hline
\end{tabular}

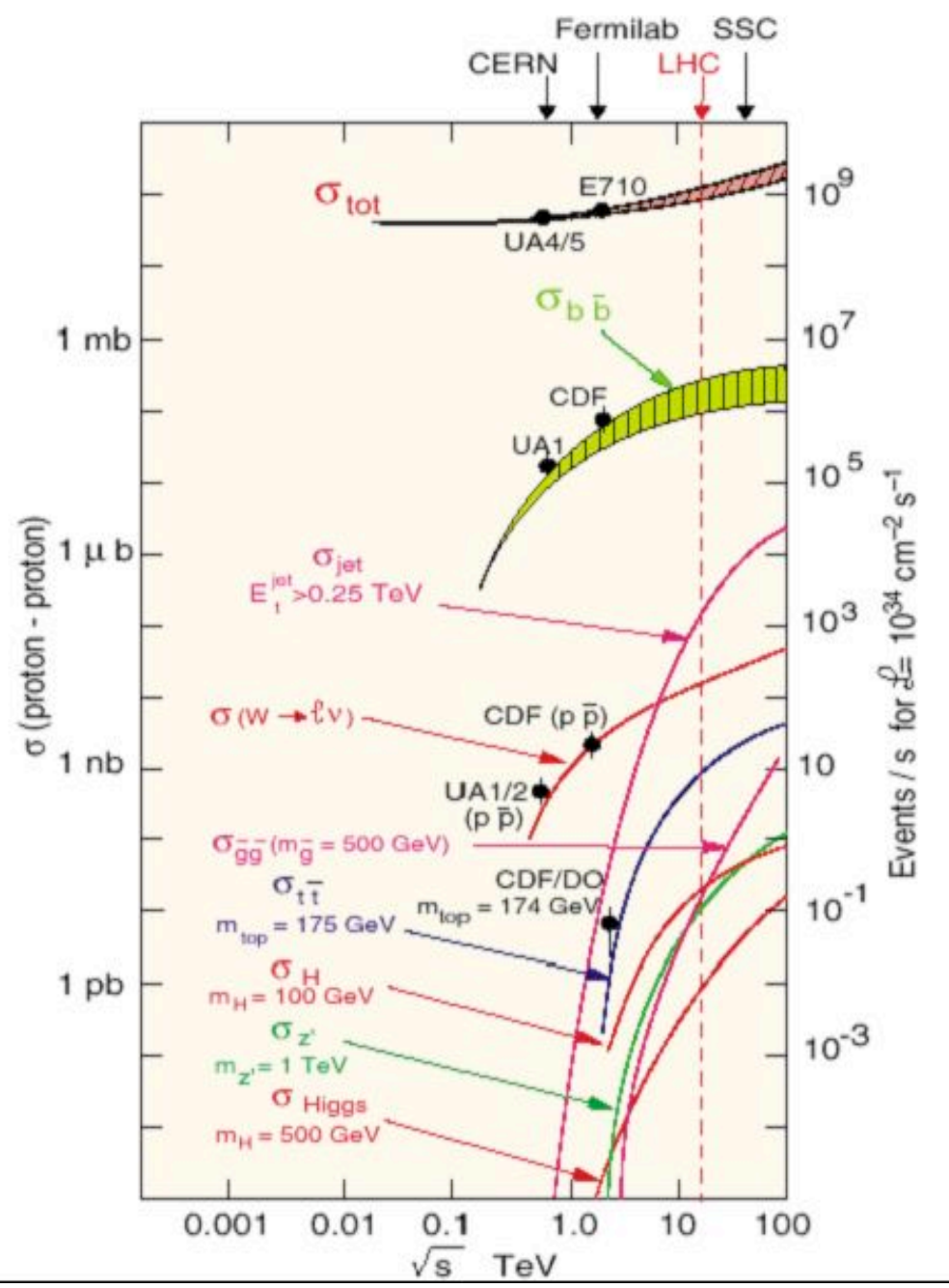

Limiting factor for $\sqrt{ } \mathrm{s}$ : Bending power needed to keep beams in $27 \mathrm{~km}$ LHC ring:

$p(T e V)=0.3 B(T) R(k m)$

With typical magnet packing factor of $\sim 70 \%$, need 1232 dipoles with $B=8.3 \mathrm{~T}$ for $7 \mathrm{TeV}$ beams 


\section{The ATLAS Detector}

4 Inner Detector (tracker):

Si pixel \& strip detectors $+T R T$;

2 T magnetic field; $|\eta|<2.5$

4 Calorimetry: highly gramular LAr EM calorimeter $(|\eta|<3.2)$; hadron calorimeter - scintillator tile; $|\eta|<4.9$

4 Muon Spectrometer: air-core toroid system; $|\eta|<2.7$

$>$ Performance:

- jet resolution: $\sigma / \mathbf{E} \approx 50 \% / \sqrt{E} \oplus 3 \%$

- $\tau$-efficiency: $\sim 50 \%$ for $R_{\text {jet }} \sim 200$

$$
\left(p_{T} \approx 60 \mathrm{GeV}\right)
$$

- missing energy: $\sigma\left(p_{x y}{ }^{\text {miss }}\right) \approx 0.46 \cdot \sqrt{ } \sum \boldsymbol{E}_{T}$ (low luminosity)

- b-tagging: $\sim 60 \%$ for $R_{\text {jet }} \sim 100$

(1)

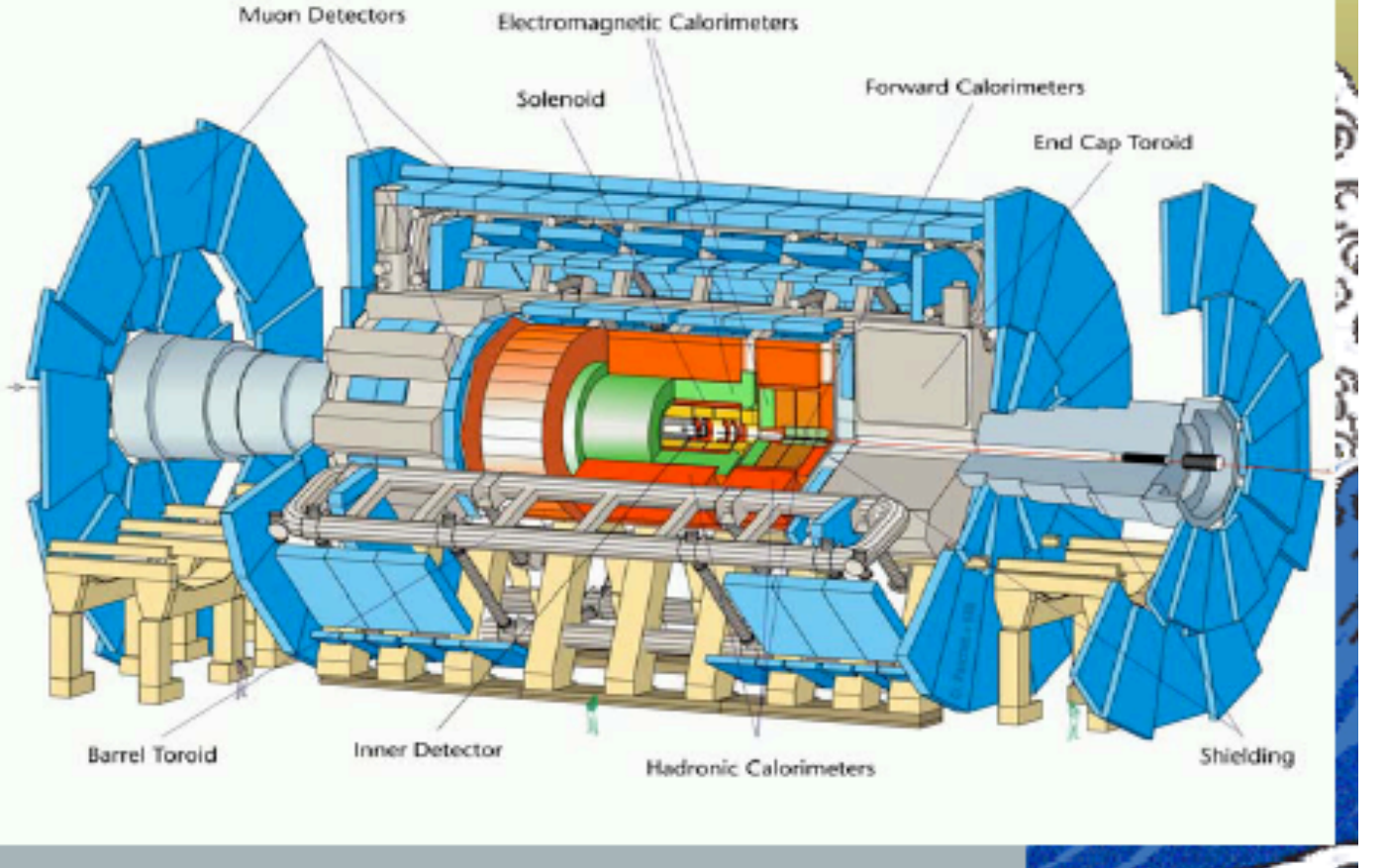

A Jet energy scale: precision of $1 \%$ $(W \rightarrow j j ; Z(l l)+$ jets $)$

ᄉ Luminosity: precision $\leq 5 \%$ (machine, optical theorem, rate of known processes )

A QCD-related measurements performed during initial period of running at low luminosity 


\section{The ATLAS Tracker}

The Inner Detector (ID) is organized into four sub-systems:

Pixels

1 removable barrel layer

2 barrel layers

4 end-cap disks on each side (0.8 $10^{8}$ channels)

Silicon Tracker (SCT)

4 barrel layers

9 end-cap wheels on each side (6 106 channels)

Transition Radiation Tracker (TRT) Axial barrel straws Radial end-cap straws 36 straws per track (4 105 channels)

Common ID items

\section{Forward SCT}

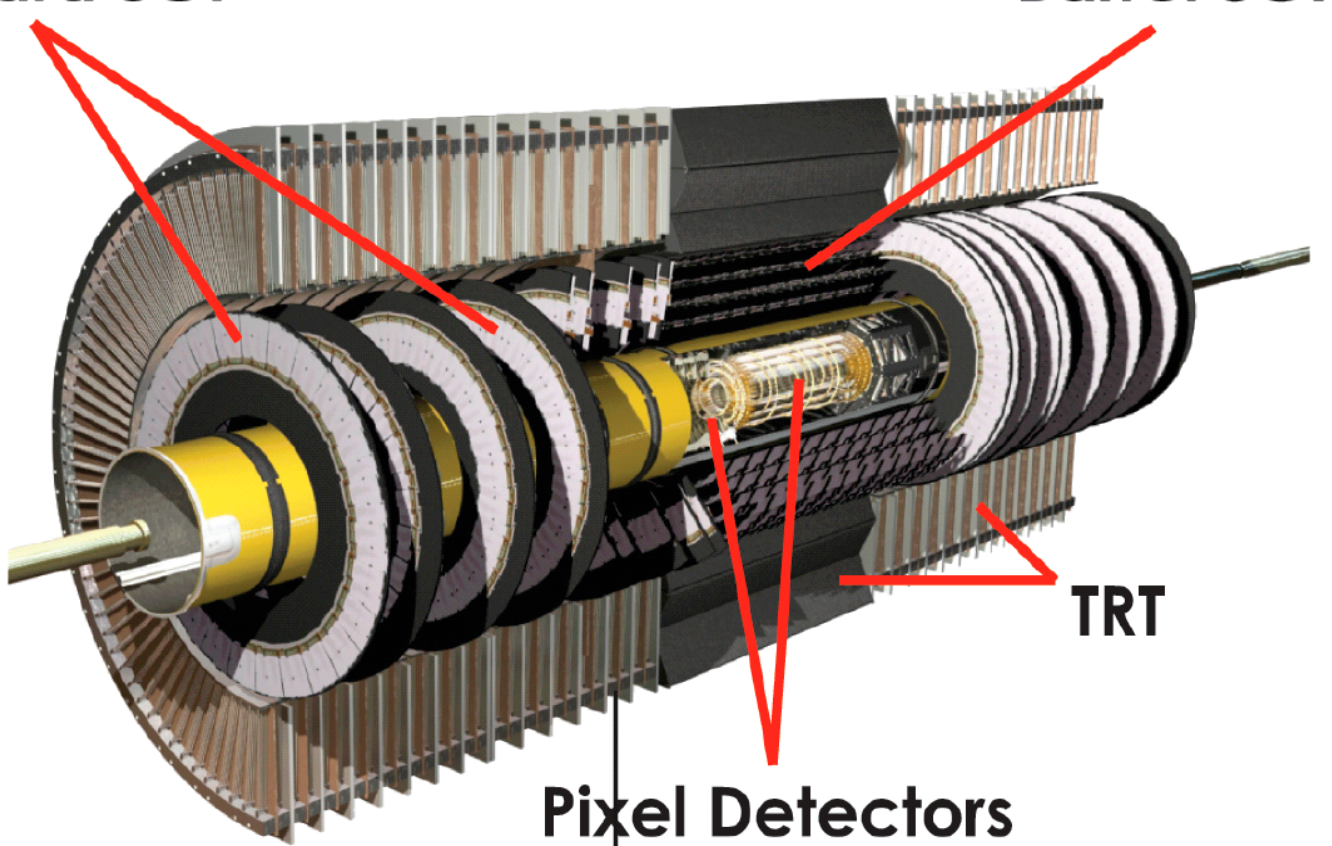

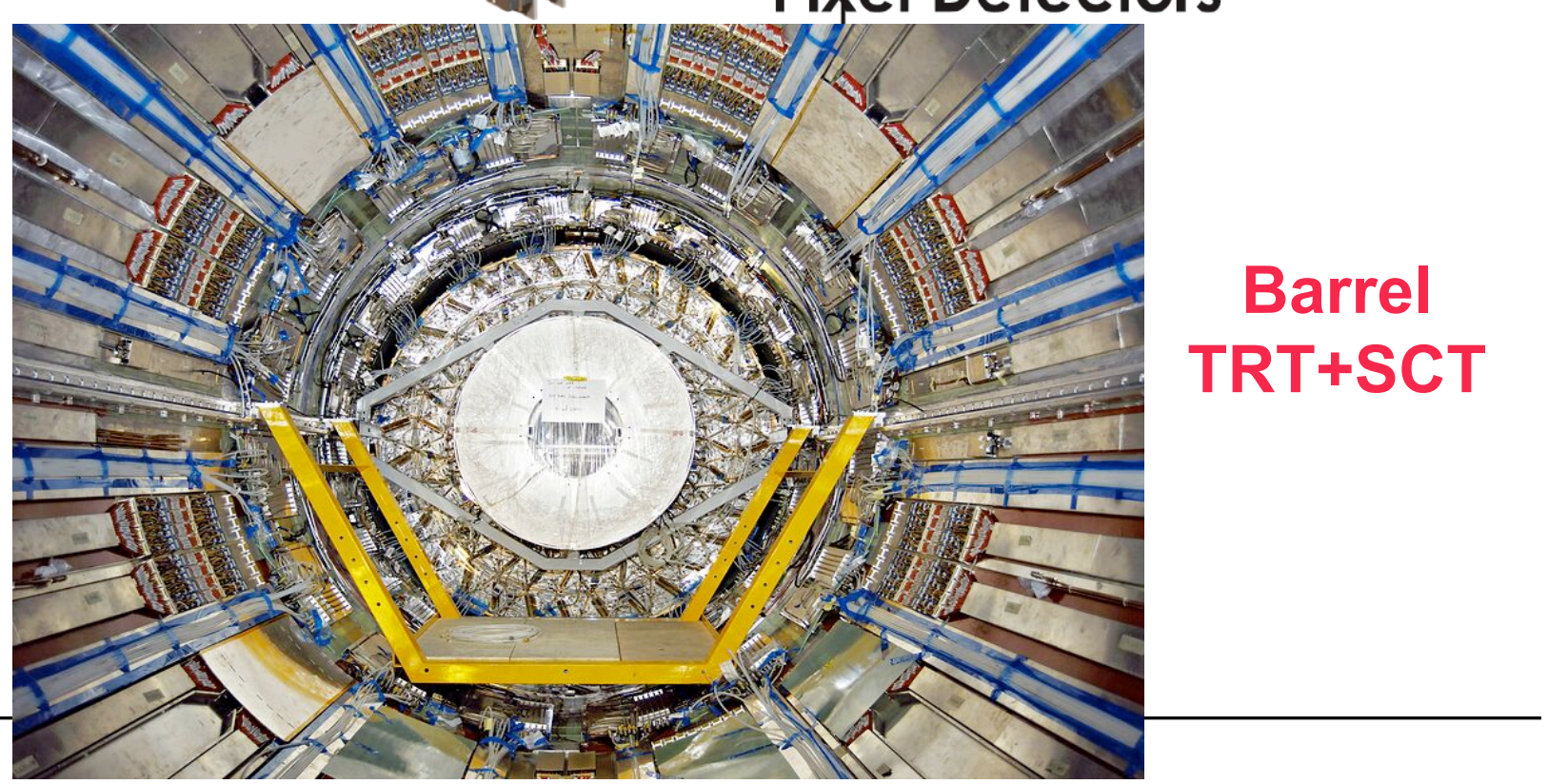




\section{$\gamma / \pi 0$ : Effects of $\gamma$ conversions}

a conversions

$\sim 30 \%$ (depending on $\eta$ ) probability for photon conversion in the ID cavity

W ID will identify and reconstruct with a $~ 80 \%$ efficiency photon conversions in the region $\mathrm{R}_{\mathrm{c}}<80 \mathrm{~cm}$ and $|\mathrm{z}|<280 \mathrm{~cm}-$ where $\sim 80 \%$ of conversions occur
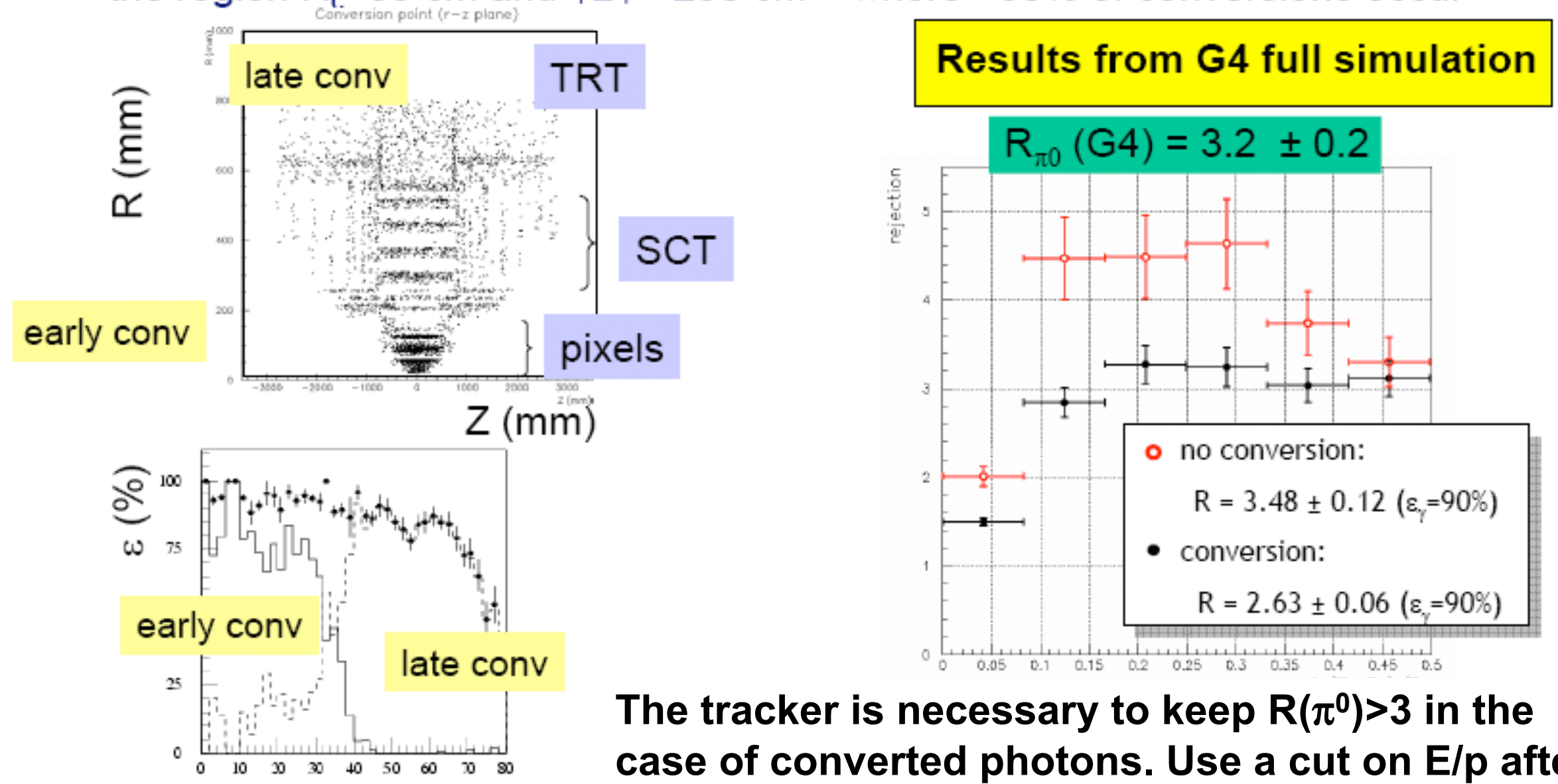

The tracker is necessary to keep $R\left(\pi^{0}\right)>3$ in the

$\mathrm{R}_{\mathrm{c}}(\mathrm{cm})$ case of converted photons. Use a cut on E/p after converted photon recovery 


\section{EM Calorimeter energy reconstruction}

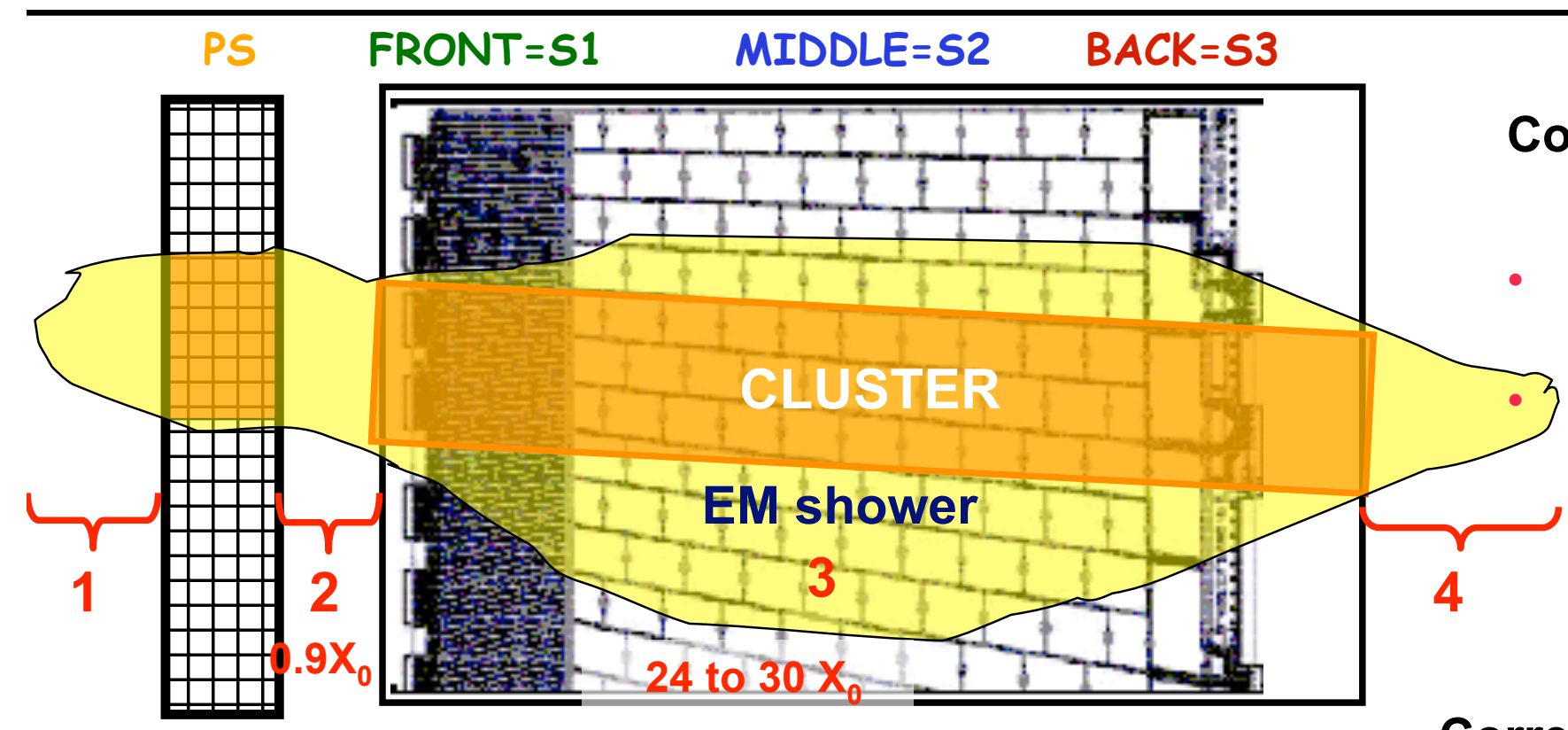

Corrections due to cluster position:

Dh (S-shape modulation)

$\pm 0.005$

Df (offset in accordion)

$\pm 0.001$

Two main clusterization methods:

Corrections for energy

- Fixed size sliding window:

$\cdot 3^{\prime} 3,3^{\prime} 7 \ldots$ cells, $2^{\text {nd }}$ sampling h'f;

-Some energy left out, especially for small sizes.

- Topological clusters:

-Variable size cluster, minimize noise impact;

-Additional splitting algorithm is also provided. losses:

1. Before PS

2. Between PS \& Calo

3. Outside cluster: depends on clustering method

4. After calorimeter:

Energy in BACK

$\mathbf{2 - 7 \%}$ overall energy correction $>7 \%$ at low energy, high $\mathrm{h}$ 


\section{Energy Calibration}

$$
E_{\text {rec }}=\lambda\left(\text { off }+w_{0} E_{0}+E_{1}+E_{2}+w_{3} E_{3}\right)
$$

The 4 coefficients are reconstructed via $c^{2}$ fit on a sample of single electrons in a $[-2 s,+3 s]$ range around the most probable value of the reconstructed energy distribution:

$$
\chi^{2}=\sum_{i}^{N} \frac{\left(E_{\text {rec }}^{i}-E_{\text {true }}^{i}\right)^{2}}{\sigma_{E}^{2}}
$$

- Simple method.

- 4-parameters, h-dependent, energy-independent.

-Weights absorb different effects and their energy dependence (offset and $\mathrm{w}_{0}$ absorb energy loss upstream the calorimeter, and between the presampler and the strips).

- It is not possible to unfold these effects. More complex approach relying on detailed understanding of $M C$ under study. 


\section{$\mathrm{H} \rightarrow$ yy Channel}

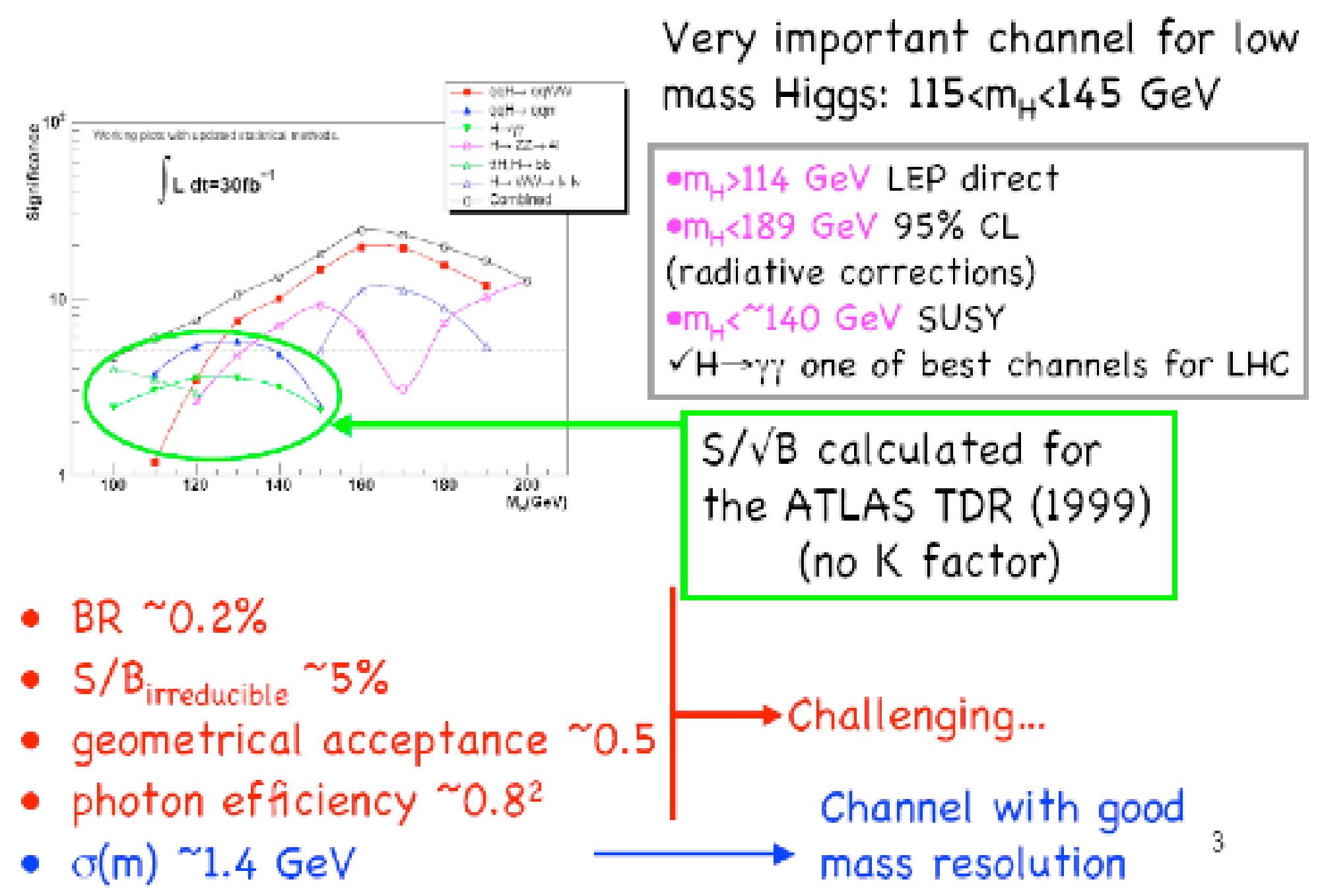




\section{Comparison of Data with NLO}

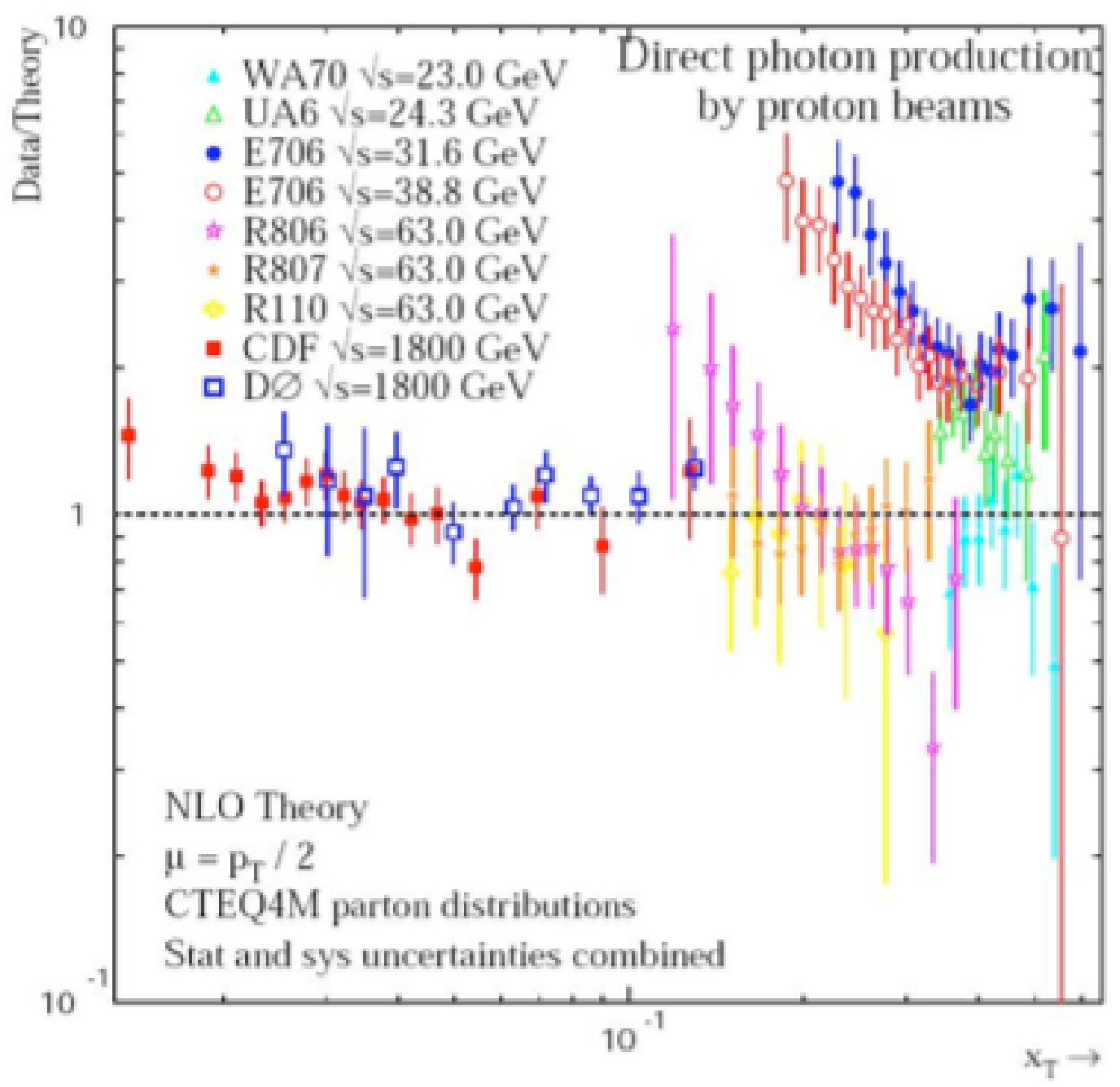




\section{Parton Luminosities and pdfs}

$$
N_{\text {events }}(p p \rightarrow X)=L_{p-p} \times p d f\left(x_{1}, x_{2}, Q^{2}\right) \times \sigma_{\text {theory }}(q, \bar{q}, g \rightarrow X)
$$

Uncertainties in p-p luminosity $( \pm 5 \%)$ and p.d.f.'s $( \pm 5 \%)$ will limit measurement uncertainties to $\pm 5 \%$ (at best).
- For high $Q^{2}$ processes LHC should be considered as a parton-parton collider instead of a p-p collider.

- Using only relative cross section measurements, might lead eventually to accuracies of $\pm 1 \%$.

\begin{tabular}{|c|c|c|}
\hline $\begin{array}{l}\quad \mathrm{q} \overline{\mathrm{q}}(\mathrm{u}, \mathrm{d}) \\
\text { (high-mass DY lepton } \\
\text { pairs and other processes } \\
\text { dominated by qवृ ) }\end{array}$ & $\begin{array}{l}\mathrm{W}^{ \pm} \text {and } \mathrm{Z} \text { leptonic } \\
\text { decays }\end{array}$ & $\begin{array}{l}\text { " precise measurements of mass and couplings; } \\
\text { " huge cross-sections }(\sim n b) ; \\
\text { " small background. } \\
\text { " x-range: } 0.0003-0.1 \\
\text { " } \pm 1 \%\end{array}$ \\
\hline \begin{tabular}{l}
\multicolumn{1}{c}{$\mathrm{g}$} \\
(high-Q⿱2 reactions \\
involving gluons)
\end{tabular} & $\gamma$-jet, Z-jet, $W^{ \pm}$-jet & $\begin{array}{l}=\gamma \text {-jet studies: } \gamma p_{\mathrm{T}}>40 \mathrm{GeV} \\
=\mathrm{x} \text {-range: } 0.0005-0.2 \\
=\gamma \text {-jet events: } \gamma p_{\mathrm{T}} \sim 10-20 \mathrm{GeV} \\
=\text { low-x: } \sim 0.0001 \\
= \pm 1 \%\end{array}$ \\
\hline $\mathrm{s}, \mathrm{c}, \mathrm{b}$ & $\gamma c, \gamma b, s g \rightarrow W c$ & $\begin{array}{l}\text { " quark flavour tagged } \gamma \text {-jet final states; } \\
=\text { use inclusive high- } p_{T} \mu \text { and b-jet identification } \\
\text { (lifetime tagging) for } \mathrm{c} \text { and } \mathrm{b} \text {; } \\
=\text { use } \mu \text { to tag c-jets; } \\
=5-10 \% \text { uncertainty for } x \text {-range: } 0.0005-0.2\end{array}$ \\
\hline
\end{tabular}

